\title{
Biophysical Reviews-Quantitative analysis of biological phenomenon
}

\author{
Damien Hall ${ }^{1}$
}

Accepted: 6 May 2020 / Published online: 4 June 2020

(C) International Union for Pure and Applied Biophysics (IUPAB) and Springer-Verlag GmbH Germany, part of Springer Nature 2020

\begin{abstract}
This Editorial first describes the articles constituting the current Issue (Volume 12 Issue 3). It then goes on to outline the formal invitation procedure for those interested in submitting a review article to the journal. The Editorial concludes by describing the nomination process for the 2021 Michèle Auger Award for Young Scientists' Independent Research.
\end{abstract}

At the time of writing, the world is in the grips of the COVID19 pandemic. Derived from a conjunction of the two Greek words "pan" (meaning all) and "demos" (meaning the people), the literal implication of a pandemic is a phenomenon affecting all people. Although the disease prevalence is differentially manifested, no populated region has been left untouched. By far, the majority of Biophysical Reviews' audience are working research scientists, engineers, and medical professionals. Another significant constituency are the undergraduate and graduate students working towards higher qualifications within these same fields. To those directly affected by illness to either themselves, or a loved one, the journal extends its sympathies and best wishes for a speedy return to health. To those not directly affected, we appreciate that you most likely are having to adapt to new social distancing rules with requirements for work/study from home. As experiments are put on hold, it may be easy to lose focus on the importance of our collective research endeavor. However, it is important to remember that it is only through society's continual investment in basic and applied research that we are able to prepare for societal emergencies (such as COVID-19), as well as provide the knowledge and tools that are (hopefully) used for the improvement of humanity's lot. Our continued scientific engagement during the current difficult times is vital for the maintenance of the international research enterprise - one of civilization's sharpest swords in its decision making arsenal.

Damien Hall

hall.damien@nitech.ac.jp; damienhall30@gmail.com

1 Department of Life Sciences and Applied Chemistry, Nagoya Institute of Technology, Gokiso Showa, Nagoya, Aichi 466-8555, Japan

\section{Issue contents}

Directly following this Editorial is the third installment of our Biophysical Reviews Meet the Editors Series, contributed by Prof. Naranamangalam Jagannathan (Jagannathan 2020). Prof. Jagannathan is a medical physicist whose life story will likely inspire younger scientists. In this scientific mini-biography, he details overcoming polio as a child and explains how this early brush with disease was the inspirational spark that launched him into a biomedical career that has seen him become a pioneer developer of several magnetic resonance imaging (MRI) diagnostic techniques. In a remarkably honest fashion, he describes the highs and lows along his career trajectory, one that culminated in him leading India's principal MRI Department at the All India Institute of Medical Sciences (AIIMS) located in New Delhi. While some scientific biographies can be a little dry, this contribution (Jagannathan 2020), very much like its two predecessors (Olson 2020; Nagayama 2020), is quite fun to read and remarkably human.

Contributed by the members of a European consortium COST Action network (Antonacci et al. 2020), the third article in this Issue reviews the latest developments in Brillouin spectroscopy for the determination of cell mechanical properties. First reported on nearly a century ago (Brillouin 1922), Brillouin scattering describes the phenomenon of light scattered from a nonrandom density fluctuation such as that caused by a traveling pressure (sound) wave. As the speed of sound in a medium is related to its mechanical properties, recording the shift in frequency of the scattered light in relation to the strength and direction of the applied acoustic pulse provides sufficient information for evaluation of localized viscoelasticity of the medium. Antonacci et al. (2020) review recent examples of how the Brillouin scattering phenomenon 
can be applied in microscopy format to interrogate and define sub-micrometer length scale mechanical heterogeneities in cultured cells. This review article potentially presages Brillouin microscopy as the next major cell imaging modality.

Arising from an international collaboration between scientists form Poland, Australia, and the Netherlands, the next article (Zapotoczny et al. 2020) reviews the truly fascinating subject of fenestrae, specialized import/export pores found within liver sinusoidal endothelial cells. Existing at the interface of the liver tissue and its blood transporting microvasculature, liver sinusoidal endothelium is a cellular monolayer that regulates exchange of nutrients between the liver and blood. Specific to the liver endothelium, fenestrae are membrane enclosed tubular pores that extend across the cell from the apical to basolateral membranes. Zapotoczny et al. (2020) review the dynamic nature of the formation, loss, and spatial localization of these 50-350-nm diameter pores concentrating on data gained using high speed or "4D" atomic force microscopy (AFM).

The next article by Yanaka et al. (2020) reviews the use of computational and structural methods to report on dynamic aspects of the immunoglobulin $\mathrm{G}$ ( $\mathrm{IgG}$ ) type antibody. Describing studies of IgG structure based on nuclear magnetic resonance (NMR), AFM, small-angle X-ray scattering, and cryo-electron microscopy, this Review provides an excellent primer on recent IgG structural literature. Going beyond facile static lock and key type conceptualizations of the antibody variable region protein interface binding to its antigenic epitope, this Review highlights the role of intrinsic conformational heterogeneity (and the dynamics of transition between these states) in the action of both IgG binding and non-binding regions. Aside from discussing the plasticity of the IgG recognition event, the authors also review the roles of specific carbohydrate modification of the $\operatorname{IgG}$ as well as describing the under-appreciated effects of higher-order oligomerization of the IgG post epitope recognition (Yanaka et al. 2020). Although functioning as a stand-alone Review, this piece was a late addition to the Special Issue dedicated to the Biophysical Society of Japan and we encourage the reader to appreciate its contribution in relation to that Issue (Komatsuzaki et al. 2020; Hall 2020a).

The sixth contribution to this Issue (Singh et al. 2020) is the journal's first review article contributed from the Jammu and Kashmir region. This article is a collaboration between a team of physicists and medical doctors reviewing the role of spectroscopic methods for the determination of the elemental and molecular composition of gall bladder and kidney stones. Split into three parts, the Review first details the presenting medical features of stone formation and the classification schema used in their diagnosis and pathology. The second part provides a review summary of the physical background to spectroscopic methods applied to stone research such as infra-red/ultra-violet spectroscopy, wavelength dispersive $\mathrm{X}$-ray fluorescence, laser-induced breakdown spectroscopy, and time of flight mass spectrometry. The third aspect of this Review discusses published findings on the composition of gall and kidney stones determined using these spectroscopic methods. By reviewing region-specific tendencies associated with stone composition, the authors note a possible dietary determinant to the etiology of stone formation and presentation (Singh et al. 2020).

The seventh article in this Issue (Kohata and Miyoshi 2020) reviews the topic of liquid-liquid phase separation (LLPS) from the perspective of these separate liquid phase regions playing a role in the storage and regulation of messenger (m), small nuclear (sn), ribosomal (r), and non-coding (nc) RNA chains. Providing a clear walk through the terminology used to define the many and varied RNA containing liquid phase regions (e.g., nucleolus, paraspeckles, nuclear speckles, Cajal bodies, PML bodies, $\mathrm{P}$ bodies, stress granules, and germ granules), Kohata and Miyoshi (2020) review evidence for the anionic polymer characteristics of RNA being a strong instigator of liquid-liquid phase separation when existing in a solution with weakly binding cationic proteins. Unlike the majority of Reviews on this topic, the authors also relate the regulation of RNA-mediated LLPS to a number of common diseases, with one prominent example being amyotrophic lateral sclerosis (ALS). This article was originally meant to be a part of the Special Issue on the Biophysical Society of Japan ${ }^{1}$ and we encourage the reader to revisit the aims and intentions of that Special Issue after reading this Review (Komatsuzaki et al. 2020; Hall 2020a).

Discussing literature taken from the "Adaptive Laboratory Evolution" (ALE) systems biology approach, the next review article deals with the subject of quantitative assessment of bacterial evolution (Horinouchi and Furusawa 2020). Readers of the journal may be familiar with simple laboratory-based evolution studies in which a single enzyme's performance is monitored for improvement through cycles of challenge (or induced mutation) and selection. The Review by Horinouchi and Furusawa (2020) discusses effective nextlevel quantitative approaches to determining organism fitness. These next-level methods incorporate multiple streams of information relating to genome sequence, transcriptome sequence, and "metabolomic" data into quantitative relations describing phenotypic advantage. Introducing innovations in both laboratory automation and formulation of analytical expressions, this review of the ALE approach signals the quantitative road ahead for identification of molecular pathways and individual components involved in evolutionary adaption. This Review was a late addition to the Special Issue dedicated to the Biophysical Society of Japan and we encourage the

\footnotetext{
${ }^{1}$ The SI Editors and I apologize to the authors of this article for our inability to process this excellent manuscript in time to make the Special Issue. This was in no part the fault of the authors.
} 
reader to appreciate its contribution in relation to that Issue (Komatsuzaki et al. 2020; Hall 2020a).

The ninth article within this Issue (Rodin 2020) reviews nuclear magnetic resonance (NMR) methodology for studying water in biopolymers (such as collagen-based gels or complex scaffolds for tissue engineering) and complex biological samples (such as pulped wood or wet soil). After reviewing some basic NMR theory (related to different modes of spin relaxation and pulsed-field gradient methods for measurement of diffusion), the author reviews a series of approaches for measuring the mobility of water and inferring its chemical and physical environment. Concentrating on parameters that can be used as correlative measures of sample quality, or alternatively, measures of sample uniformity, Rodin (2020) describes both practical examples (such as the assessment of heterogeneity in proton spin-spin $\left(\mathrm{T}_{2}\right)$ relaxation constants in spruce pine samples) and simulated results (such as correlation analysis of the diagonal components of the diffusion tensor obtained from orthogonal pulse-field gradient diffusion experiments). This Review demonstrates how modern NMR methods can be used to practical advantage in sample characterization.

The penultimate contribution, resulting from an international collaboration between scientists from Algeria, India, and Iran, is concerned with the use of quantum dots (QDs) in nanomedicine (Nikazar et al. 2020). Essentially spherical semiconductors, the electronic transition between valence and conduction bands in QDs is greatly affected by their nano-scale radius of curvature (in the range of 1-10 nm). This nanoscale aspect of the physics allows them to fluoresce at visible wavelengths, in a manner that can be "tuned" upon changing either the semiconductor material or the size of the quantum dot. Appropriately modified, these QDs are used in nanomedicine for bioimaging and magic bullet therapy, although one major drawback (jointly stemming from their semiconductor composition and high specific surface area to volume ratio) is their significant cytotoxicity. This Review by Nikazar et al. (2020) reviews a large number of studies for the factors that both produce and mitigate such toxicity. An interesting feature of this Review is its additional examination of toxicity at the environmental level as might be important for the case of QD pollution arising from its indiscriminate release.

Second messengers are intracellular signaling molecules released within the cell after membrane receptors bind an extracellular signaling molecule (which constitutes the first messenger). The final article of Issue 3 reviews the multiple signal transduction roles of a ubiquitous bacterial second messenger known as cyclic di-guanosine monophosphate (c-di-GMP) (Petchiappan et al. 2020). Among the many roles of c-diGMP, it regulates the transition between the bacterial swimming "planktonic" stage and the non-motile biofilm growth stage. Aside from describing these and other functional roles, this Review discusses recently developed bioanalytical tools for the quantitative measurement and regulation of c-di-GMP levels within the cell. With a focus on fluorescence methods, the authors describe a series of c-di-GMP biosensors based on c-di-GMP binding riboswitches and Förster resonance energy transfer (FRET) constructs involving c-di-GMP binding proteins. Petchiappan et al. (2020) synthesize the available information to present a spatiotemporal picture of c-di-GMP's sources and sinks within the cell.

\section{Engaging with Biophysical Reviews}

The journal has previously published on the benefits (and disadvantages) of writing a Review (Hall 2019a). One of the noted negative features of Review preparation was the time spent away from generating primary research results. For many researchers currently coping with the COVID-19 lockdown, this particular negative aspect is no longer present. For those interested in writing a Review, I describe the general process for engaging with Biophysical Reviews with the intention of writing, submitting, publishing, and promoting your review article.

(i.) Consultation with an Editorial Board Member: Biophysical Reviews principally operates in an invitation to publish based manner through which review articles are commissioned by Members of the Editorial Board and coordinated for publication within a particular Issue. However, if you have a proposal for an article, you are welcome to make direct contact with the Chief Editor, or alternatively, your local Editorial Board Member. Typically, during this negotiation phase, you will be asked to provide a manuscript title, a prospective list of authors, and a short abstract. A provisional submission date will be agreed upon and the target Issue set upon the condition of the manuscript being accepted after review by two experts in the field (with necessary revisions being satisfactorily completed).

(ii.) Receiving an invitation to publish: Once the topic has been judged appropriate for the journal, you will receive an official offer from the commissioning Editorial Board Member asking you to commit to submitting your article by a particular date (agreed upon during the consultation phase). Attached to this email will be two documents, the first summarizing the journal website and submission process, the second an "instructions for authors" detailing necessary referencing formats and figure requirements. This email letter will contain within the cc line the email addresses of the Chief Editor, the Springer-Nature journal Manager and a Springer Editorial Assistant. You will be asked to reply to this email agreeing to the proposed topic and timing, including all respondents in your reply.

(iii.) Preparing and submitting your Review: The agreed upon submission deadline provides for eight weeks to have your manuscript reviewed and requested revisions completed. Typically, you will receive two expert critiques of your manuscript within 4 
weeks. If the reviews are favorable, the journal will then request that the necessary revisions be made within 2 weeks of receipt of the initial critique. Upon receiving the revised manuscript, it will (if necessary) be sent back to the reviewers for a second review, after which a decision will be conferred by the handling Editor. If accepted, your manuscript will then enter into production. The first stage of production involves copyright transfer and decision on article payment by the corresponding author. As mentioned previously, the journal operates a hybrid two-track option with regard to copyright transfer and payment (Hall 2019b). The first option is completely free but involves the review article remaining behind a pay firewall for a period of 6 to 12 months. The second option involves payment of an open access fee which removes the payment firewall for your article. Both options produce an excellent outcome and the author is entirely free to choose which payment pathway to proceed along. The second stage of production involves the desktop publishing of your article by the Springer-Nature professional staff. Typically, the corresponding author will receive online page proofs of the article within 2 weeks of resolution of the copyright transfer agreement. This stage represents the last chance for minor corrections to be made.

(iv.) Post-publication promotion of your article: Following publication, the corresponding author will receive a request to participate in a social media promotion particular to the Issue in which their article appears. The participation process involves (a) application for a Springer SharedIt ${ }^{\mathrm{TM}}$ link for the published article, (b) filling out a pro forma template with information on your article such as title, summary, and professional contact information. As discussed previously, the SharedIt ${ }^{\mathrm{TM}}$ program provides a copyright-free means for unlimited posting and sharing of your article on any form of social media, personal or professional website (Hall 2017). Based around the SharedIt ${ }^{\mathrm{TM}}$ program, the journal uses the information provided by the author(s) in two ways. The first way involves the posting of a 4-minute video summary of the Issue contents to the Biophysical Reviews' YouTube ${ }^{\mathrm{TM}}$ channel. Set to a variable genre of musical accompaniment, the Issue summary represents a very modern method for skimming the Issue contents and is a feature currently unique to Biophysical Reviews. The journal's YouTube $^{\mathrm{TM}}$ channel can be found here:

https://www.youtube.com/channel/UCzG_ 5MWmnrB2UBibtxs2DuA

The second means of using the article summary information involves its individual "tweeting" with cross posting of the information contained on both the Biophysical Reviews' YouTube $^{\mathrm{TM}}$ channel and official Springer website. The Biophysical Review's Twitter ${ }^{\mathrm{TM}}$ account can be found at the following address:

@BiophysicalRev1

Both the YouTube $^{\mathrm{TM}}$ and Twitter $^{\mathrm{TM}}$ accounts are intentionally non-controversial (i.e., YouTube ${ }^{\mathrm{TM}}$ comments are disabled and only Twitter $^{\mathrm{TM}}$ Followers are able to post mentions). Nevertheless, the content on both of these platforms can be used by authors for promotion of their work through reposting and retweeting by either themselves or their Institutional media representatives. The journal website monitors these alternative metrics under the altimetric banner associated with each online article. Having the official imprimatur of the journal promoting your work may help to add some social media "kudos" to your own (or a student's) retweeting of the importance of your published work. Indeed, the leveraging of this journal provided advantage by the author is a major goal of the journal's social media program and we highly encourage our authors to engage in these activities.

\section{The Michèle Auger Award for 2021}

The close of 2018 brought with it the sad news of the death of Biophysical Reviews' Editorial Board Member, Prof. Michèle Auger (IUPAB 2019). With the kind support of SpringerNature Corporation, Biophysical Reviews announced the creation of a perpetual memorial award in honor of Michèle's life and service. The "Michèle Auger Award for Young Scientists' Independent Research" is to be granted each year to a single candidate performing biophysical research, who is under 40 years of age at the time of application. The award consists of a plaque, a free personal subscription to the journal, and an invitation to submit a single author review article to be published in Biophysical Reviews. The published Review will carry a short foreword about the life of Prof. Michèle Auger, along with her work associated with teaching and training the next generation of biophysical scientists.

Nominations for the 2021 award can be made in the form of a candidate's one-page curriculum vitae, along with five original manuscripts, to be submitted by email to either the Chief Editor or any one of the Biophysical Review's Executive Editors, prior to October 31st. Judging will be carried out by a special committee assembled from the Editorial Board, with this assembly taking place after the submission deadline. The winner will be announced in late December, with the winner's single-author Review to be published in the following year.

The 2020 Michèle Auger Award proved quite popular with over 28 applications received by the deadline close in late 2019. The winner of the inaugural award for 2020 was Dr. Alexandra Zidovska. Alexandra is currently an NSF Career Fellow and Assistant Professor at the Center for Soft Matter Research, Department of Physics, New York University. More about her research can be found at her laboratory home page.

https://as.nyu.edu/content/nyu-as/as/faculty/alexandrazidovska.html

Alexandra's review article will appear as the lead article of Volume 12 Issue 5 (to be published mid-October 2020). In line with Michèle's interest in promoting young scientist's 
research work, the journal encourages nominations for the coming 2021 competition.

\section{Concluding remarks}

Due to its association with the International Union for Pure and Applied Biophysics (IUPAB) Biophysical Reviews is genuinely interested in fostering international research and education in biophysical research. Some of the actions of Biophysical Reviews in furthering these goals can be appreciated by reading the end of year and year opening summaries (Hall 2019c; Hall 2020b). Volume 12 Issue 2 was the first experiment for the journal in partnering with a national biophysical society to assist in promoting the collective work of its members on a world platform. I would again like to thank the Biophysical Society of Japan and the Special Issue Editors (Komatsuzaki et al. 2020) for their enthusiastic and wholehearted cooperation with that endeavor. That Special Issue is to date the largest ever run by the journal and was concluded under genuinely challenging circumstances. In regard to this last point, I would like to acknowledge the extraordinary dedication of the journal's professional staff working at the Springer-Nature offices in the Philippines.

As a final note, I would like to express my personal thanks to the Nagoya Institute of Technology (NITech) for facilitating my return to Japan in March. With over a 115-year history, NITech is a specialist science, engineering, and technology university located within Nagoya - the capital city of Aichi prefecture in central Japan. Similar to the growing worldwide trend for such activities, NITech has recently made efforts to attract overseas students into its graduate programs as well as provide its domestic students greater exposure to international research venues. More can be found out about the Nagoya Institute of Technology here (NITech 2020).

Acknowledgments Thanks to Dr. Meran Lloyd-Owen for comments on an early draft of the current manuscript. I acknowledge the Nagoya Institute of Technology for an appointment to their "Visiting International Scientist" program carried out at the Department of Life Science and Applied Chemistry. All opinions expressed in this paper are the author's and do not necessarily reflect the policies and views of the Nagoya Institute of Technology.

\section{References}

Antonacci G, Beck T, Bilenca A, Czarske J, Elsayad K, Guck J, Kim K, Krug B, Palombo F, Prevedel R, Scarcelli G (2020) Recent progress and current opinions in Brillouin microscopy for life science application. Biophys Rev 12(3) Current Issue

Brillouin L (1922) Diffusion de la lumière et des rayons X par un corps transparent homogène. Ann Phys 9:88-122

Hall D (2017) Innovations in publication: free sharing of all biophysical reviews' content. Biophys Rev 9(2):67-69

Hall D (2019a) The benefits of writing a review. Biophys Rev 11(5):663666

Hall D (2019b) Biophysical reviews enters the online world, provides a focus on biophysics in Asia. Biophys Rev 11(3):249-250

Hall D (2019c) 2019 - a year in biophysical reviews. Biophys Rev 11(6): $1-7$

Hall D (2020a) Biophysical reviews' national biophysical society partnership program. Biophys Rev 12(2):187-192

Hall D (2020b) A new decade for biophysical reviews and a look into the future of biophysics. Biophys Rev 12(1):1-7

Horinouchi T, Furusawa C (2020) Understanding metabolic adaptation by using bacterial laboratory evolution and transomics analysis. Biophys Rev 12(3) Current Issue

IUPAB (2019) Newsletter, issue 70 (February). http://iupab.org/2018/11/ 06/obituary-michele-auger-former-iupab-councilor/

Jagannathan NR (2020) Biophysical reviews' 'meet the editors series' - a profile of Naranamangalam R Jagannathan. Biophys Rev 12(3) Current Issue

Kohata K, Miyoshi D (2020) RNA phase separation-mediated direction of molecular trafficking under conditions of molecular crowding. Biophys Rev 12(3) Current Issue

Komatsuzaki T, Nakamura H, Tame J, Yanaka S, Nagai T, Nagayama K (2020) Editorial for the special issue of Biophy rev focused on the biophysical Society of Japan with select scientific content from the 57th BSJ annual meeting, Miyazaki, Japan. Biophys Rev 12(2): 183-185

Nagayama K (2020) Biophy rev' "meet the editors series" - a profile of Kuniaki Nagayama: encounters and leaps in a transborder journey through biophysics. Biophys Rev 12(2):193-199

Nikazar S, Sivasankarapillai VS, Rahdar A, Gasmi S, Anumol PS, Shanavas MS (2020) Revisiting the cytotoxicity of quantum dots: an in-depth overview. Biophys Rev 12(3) Current Issue

NITech (2020) https://www.nitech.ac.jp/eng/abou

Olson WK (2020) Biophysical reviews' "meet the editors series"- a profile of Wilma K. Olson Biophys Rev 12(1):9-12

Petchiappan A, Naik SY, Chatterji D (2020) Tracking the homeostasis of second messenger cyclic-di-GMP in bacteria. Biophys Rev 12(3) Current issue

Rodin VV (2020) NMR techniques in studying water in biotechnological systems. Biophys Rev 12(3) Current issue

Singh VK, Jaswal BS, Sharma J, Rai PK (2020) Analysis of stones formed in the human gall bladder and kidney using advanced spectroscopic techniques. Biophys Rev 12(3) Current issue

Yanaka S, Yogo R, Kato K (2020) Biophysical characterization of dynamic structures of immunoglobulin G. Biophys Rev 12(3) Current issue

Zapotoczny B, Braet F, Wisse E, Lekka M, Szymoński M (2020) Biophysical nanocharacterization of liver sinusoidal endothelial cells through atomic force microscopy. Biophys Rev 12(3) Current issue

Publisher's note Springer Nature remains neutral with regard to jurisdictional claims in published maps and institutional affiliations. 